\title{
Laboratory Test Result
}

National Cancer Institute

\section{Source}

National Cancer Institute. Laboratory Test Result. NCI Thesaurus. Code C36292.

The outcome of a laboratory test. 